\title{
Population Pharmacokinetic Analysis of Trastuzumab using the Mixed-Effect Modeling Function-NLME
}

\author{
Tomoo FUNAKI* and Mari SHIOMI* \\ (Received on October 17, 2001) \\ * Clinical Pharmacology Group, Nippon Roche K. K. \\ 2-6-1 Shiba, Minato-ku, Tokyo 105-8532, Japan
}

\begin{abstract}
Nonlinear Mixed-Effects Methods for S-PLUS (Mixed-effects modeling function-version 3.3 NLME) have been reported. A zero-order infusion model was created for NLME in this study. Data from the literature for trastuzumab were used for analysis with the zero-order infusion model for NLME ; data for single as well as multiple administration ( 1 q per week) studies were available for doses of $1,2,4$ and $8 \mathrm{mg} / \mathrm{kg}$.

The two-compartment model was better than the one-compartment model in describing the disposition of trastuzumab, for both single and multiple administration. In single-dose fitting, the predicted concentrations agreed well with the observed concentrations in the one-compartment model as well as in the twocompartment model. However, in multiple-dose fitting, the predicted concentrations slightly underestimated the observed concentrations at higher doses; this was considered evidence of the nonlinear pharmacokinetics of trastuzumab or a problem with the estimation of half-life.
\end{abstract}

Key words : NLME, S-PLUS, trastuzumab, shed antigen

\section{Introduction}

Population pharmacokinetic/pharmacodynamic $(\mathrm{PK} / \mathrm{PD})$ model analysis in drug development has recently been increasing to assure qualitative and efficient conduct of clinical trials. The impact of population $\mathrm{PK} / \mathrm{PD}$ analyses on the drug development process through the experience at ParkeDavis has been reported ${ }^{11}$.

Trastuzumab is a recombinant humanized monoclonal antibody with high affinity for the HER2 protein, and inhibits the growth of breast cancer cells overexpressing HER2. The pharmacokinetics of trastuzumab in patients with HER2/neuoverexpressing metastatic breast cancer were reported by Tokuda et $\mathrm{al}^{2)}$, with half-life $\left(t_{1 / 2}\right)$ estimated by model-independent analysis increasing with an increase in dose. However, the final sampling points were the same for each dose in their analysis, i. e. the serum concentration profiles were truncated at the final sampling point. This truncat-

*日本ロシュ株式会社医薬開発本部臨床薬理グループ

干 105-8532 東京都港区芝 2-6-1 ed profile might partially explain the reason for dose-dependence of $t_{1 / 2}$. In order to test this possibility, population pharmacokinetics were examined.

Software for population pharmacokinetics and pharmacodynamics was summarized by Aarons ${ }^{3)}$, who reported NONMEM as the most widely used program for the analysis of population pharmacokinetic and pharmacodynamic data. On the other hand, statistical graphics in pharmacokinetics and pharmacodynamics have been reported ${ }^{4}$. Statistical graphs may comprise a part of an exploratory population data analysis. NLME was reported by Pinheiro and Bates $^{5}$ for nonlinear mixed-effects models, in which NLME was implemented in the S-PLUS software ${ }^{6}$. NLME provides summary statistics and graphical analysis via S-PLUS, but NONMEM does not provide such summary statistics and its graphical analysis uses a printer plot ${ }^{3)}$. Although Xpose ${ }^{7}$ provides graphical analysis with NONMEM output, the combination of NLME and S-PLUS might be much easier for exploratory population data analysis. Recently, a library of pharmacokinetic models, i.e. pklibrary, has 
become available ${ }^{8)}$; individuals who are not familiar with $\mathrm{S}$ programming can therefore also use NLME for population pharmacokinetic analysis.

The aim of the present study was to create a zero-order infusion model for NLME trastuzumab analysis and to examine the possibility of nonlinearity of the pharmacokinetics of trastuzumab. The effect of shed antigen on the pharmacokinetics of trastuzumab was also studied.

\section{Materials and Methods}

\section{Data}

The original data used for this analysis can be found elsewhere ${ }^{2}$. Data from a total of 18 patients with metastatic breast cancer refractory to chemotherapy were used. The doses were 1, 2, 4 and $8 \mathrm{mg} /$ $\mathrm{kg}$ of trastuzumab delivered as a $90 \mathrm{~min}$ intravenous infusion. The first dose was followed in 3 weeks by nine weekly doses.

\section{Model}

Serum concentration following zero-order infusion in a one-compartment model can be described as in equation 1 .

$$
\begin{aligned}
& \text { if time } \leq \mathrm{t}_{\mathrm{1}} \text { tstar }=\text { time else } \mathrm{tstar}=\mathrm{t}_{\mathrm{i}} \\
& \mathrm{C}=\frac{\mathrm{k}_{0}}{\mathrm{~V} \cdot \mathrm{k}}\left(1-\mathrm{e}^{-\mathrm{k} \cdot \mathrm{tstar}}\right) \cdot \mathrm{e}^{-\mathrm{k} \cdot(\text { time-tstar })}
\end{aligned}
$$

where $t_{1}$ is infusion time, $k_{0}$ is zero-order infusion rate constant, $\mathrm{V}$ is volume of distribution and $\mathrm{k}$ is the elimination rate constant. The serum concentration following zero-order infusion in a two-compartment model can be described as in equation 2 .

$$
\begin{aligned}
& \text { if time } \leq \mathrm{t}_{1} \text { tstar }=\text { time else tstar }=\mathrm{t}_{\mathrm{i}} \\
& \begin{aligned}
\mathrm{C} & =\frac{\mathrm{k}_{0} \cdot\left(\mathrm{k}_{21}-\alpha\right) \cdot\left(1-\mathrm{e}^{-\alpha \cdot \mathrm{tstar}}\right)}{\mathrm{V}_{1} \cdot \alpha \cdot(\beta-\alpha)} \cdot \mathrm{e}^{-\alpha \cdot(\text { time-tstar) }} \\
& +\frac{\mathrm{k}_{0} \cdot\left(\mathrm{k}_{21}-\beta\right) \cdot\left(1-\mathrm{e}^{-\beta \cdot \mathrm{tstar}}\right)}{\mathrm{V}_{1} \cdot \beta \cdot(\alpha-\beta)} \cdot \mathrm{e}^{-\beta \cdot(\text { time-tstar) }}
\end{aligned}
\end{aligned}
$$

where $\alpha$ is slope of $\alpha$-phase, $\beta$ is the slope of $\beta$-phase, $V_{1}$ is volume of distribution in compartment 1 and $k_{21}$ is the transport rate constant from compartment 2 to compartment 1 . The serum concentration following zero-order infusion in a three- compartment model can be described as in equation 3.

$$
\begin{aligned}
& \text { if time } \leq \mathrm{t}_{1} \mathrm{tstar}=\text { time else tstar }=\mathrm{t}_{1} \\
& \begin{aligned}
\mathrm{C} & =\frac{\mathrm{k}_{0} \cdot\left(\mathrm{k}_{21}-\alpha\right) \cdot\left(\mathrm{k}_{31}-\alpha\right) \cdot\left(1-\mathrm{e}^{-\alpha \cdot \mathrm{tstar}}\right)}{\mathrm{V}_{1} \cdot \alpha \cdot(\gamma-\alpha) \cdot(\beta-\alpha)} \cdot \mathrm{e}^{-\alpha \cdot(\text { time}-\mathrm{tstar})} \\
& +\frac{\mathrm{k}_{0} \cdot\left(\mathrm{k}_{21}-\beta\right) \cdot\left(\mathrm{k}_{31}-\beta\right) \cdot\left(1-\mathrm{e}^{-\beta \cdot \mathrm{tstar})}\right)}{\mathrm{V}_{1} \cdot \beta \cdot(\gamma-\beta) \cdot(\alpha-\beta)} \cdot \mathrm{e}^{-\beta \cdot(\text { time-tstar) }} \\
& +\frac{\mathrm{k}_{0} \cdot\left(\mathrm{k}_{21}-\gamma\right) \cdot\left(\mathrm{k}_{31}-\gamma\right) \cdot\left(1-\mathrm{e}^{-\gamma \cdot \mathrm{tstar})}\right)}{\mathrm{V}_{1} \cdot \gamma \cdot(\alpha-\gamma) \cdot(\beta-\gamma)} \cdot \mathrm{e}^{-\gamma \cdot(\text { time-tstar) }}
\end{aligned}
\end{aligned}
$$

where $\gamma$ is the slope of $\gamma$-phase and $k_{31}$ is the transport rate constant from compartment 3 to compartment 1.

\section{Software}

NLME version $3.3^{5)}$ was used for population pharmacokinetic analysis. S-PLUS $2000^{6}$ ) was used to run NLME. Visual $\mathrm{C}^{\left.++6.0^{9}\right)}$ was used to make a $\mathrm{C}$ model for NLME.

\section{Results and Discussion}

\section{Single-dose administration}

Data with metastatic breast cancer refractory to chemotherapy following single administration were fitted to one- and two-compartment models with zero-order infusion as shown in equations 1 and 2 . The doses used were $1,2,4$ and $8 \mathrm{mg} / \mathrm{kg}$ of trastuzumab delivered as a $90 \mathrm{~min}$ intravenous infusion. NLME fitting in the two-compartment model was slightly better than that in the one-compartment model ; the AIC for the one-compartment model was 4944 and that for the two-compartment model was 4883 (Table 1). Thus, the two-compartment model was better than the one-compartment model for describing the disposition of trastuzumab $(p<$ 0.0001 ) in the case of single administration. A comparison between values predicted by the twocompartment model and observed values is shown in Fig. 1. The predicted values agreed well with the observed values. NLME fitting using the threecompartment model failed due to problems with identification of the three phases. We therefore concluded that the two-compartment model is more appropriate than the one-compartment model for describing the disposition of trastuzumab following 
Table 1 Summary of NLME Analysis of Single-administration Data using a Two-compartment Model

\begin{tabular}{lrllll}
\hline \multicolumn{2}{c}{ Fixed effects : } & & & \\
& Value & Std.Error ${ }^{\text {a) }}$ & $\mathrm{DF}^{\text {b) }}$ & t-value & p-value \\
$\mathrm{V}_{1}$ & 51.484 & 2.3575 & 201 & 21.838 & $<.0001$ \\
$\alpha$ & 0.395 & 0.1108 & 201 & 3.568 & 0.0005 \\
$\beta$ & 0.047 & 0.0222 & 201 & 2.117 & 0.0355 \\
$\mathrm{k}_{21}$ & 0.192 & 0.1017 & 201 & 1.888 & 0.0605
\end{tabular}

Random effects :

$\begin{array}{lllll} & \mathrm{V}_{1} & \alpha & \beta & \text { Residual } \\ \text { StdDev } & 6.8349 & 0.027712 & 0.00005495 & 13196\end{array}$

AIC BIC $\log L i k$

$\begin{array}{lll}4883 & 4910.3 & -2433.5\end{array}$

Number of Observations : 222

Number of Groups : 18

a) Parameter's standard error b) Degree of freedom

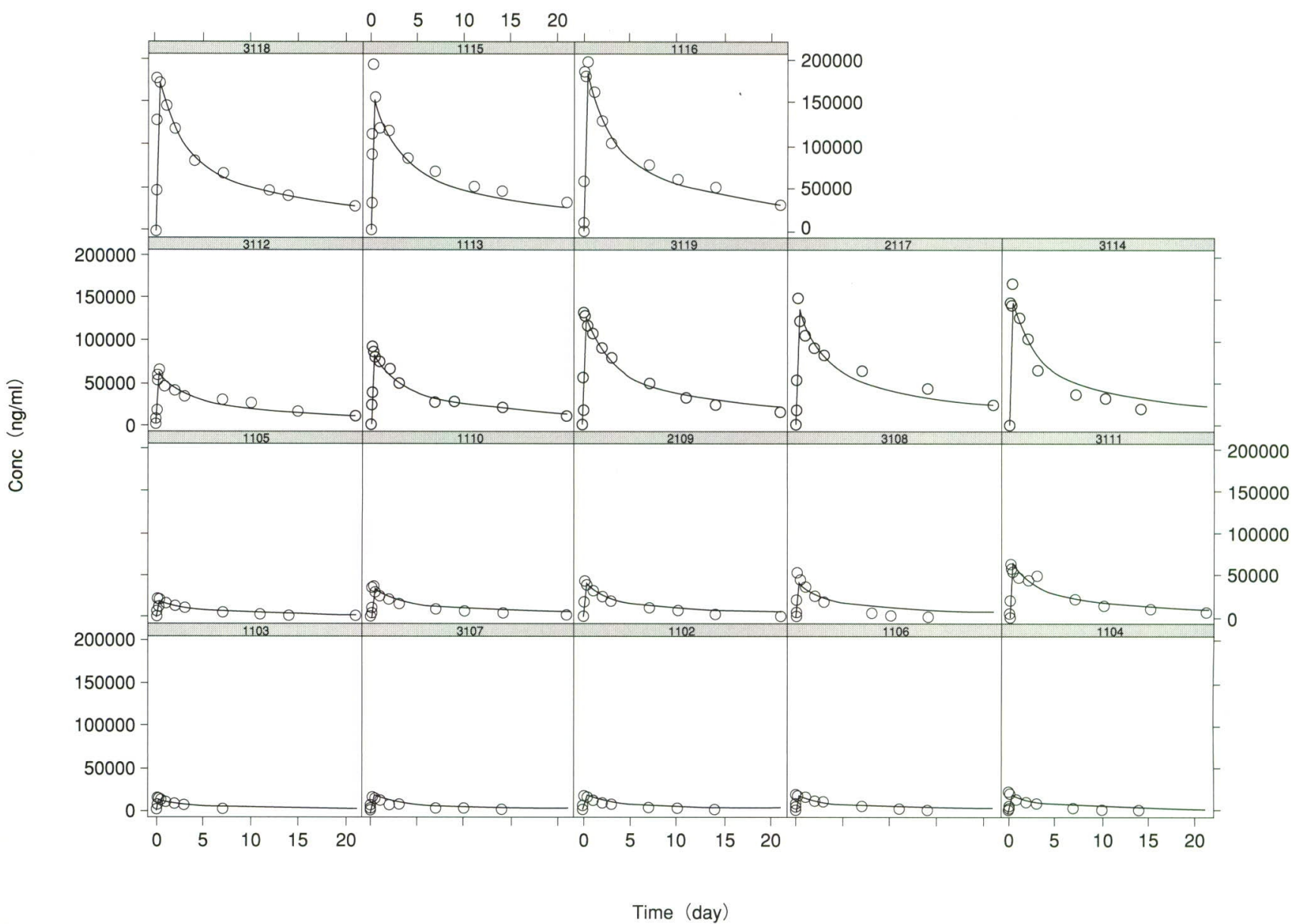

Fig. 1 Single dose data : Comparison between values predicted by the two-compartment model (solid line) and observed values (open circles). Doses : $1 \quad(n=6 ; I D=1103,1102,1104,1105,1106,3107), 2 \quad(n=3 ; I D=1110$, $2109,3108), 4 \quad(n=3 ; I D=3111,3112,1113), 8 \quad(n=6 ; I D=1115,1116,2117,3114,3118,3119) \mathrm{mg} / \mathrm{kg}$ 


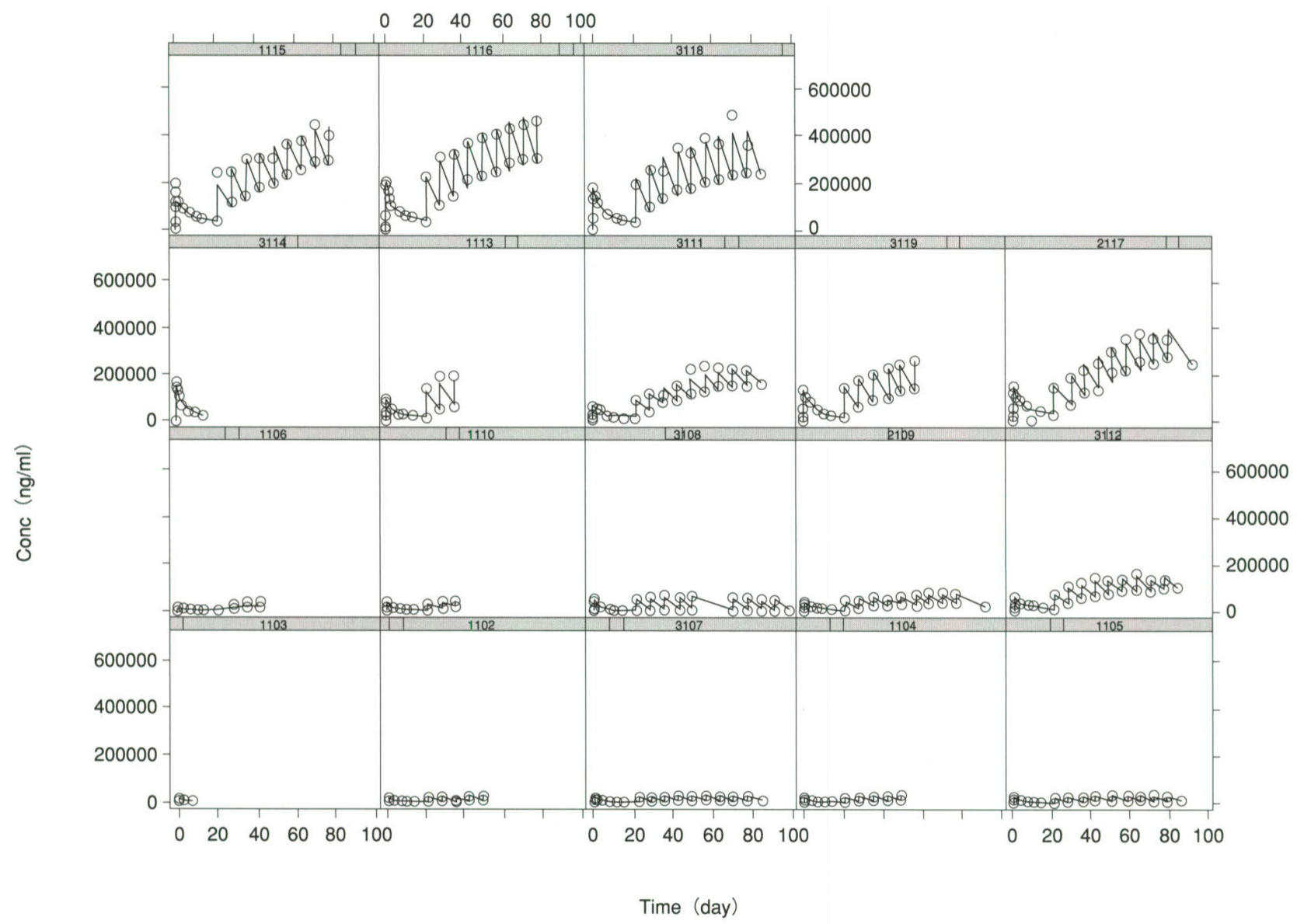

Fig. 2 Multiple dose administration following single dose administration : Comparison between values predicted (solid line) by the two-compartmental model and observed values (open circles). Doses : $1(n=6 ; \mathrm{ID}=$ $1103,1102,1104,1105,1106,3107), 2 \quad(n=3 ; I D=1110,2109,3108), 4 \quad(n=3 ; I D=3111,3112,1113), 8 \quad(n=6$; $\mathrm{ID}=1115,1116,2117,3114,3118,3119) \mathrm{mg} / \mathrm{kg}$

single-dose administration.

Both NONMEM and NLME compute the approximate maximum likelihood estimates by expansion of the nonlinear compartmental modeling function to linear expansion about random effect beta $_{1}=0$ in NONMEM and approximation by linearization about the current best estimate of beta ${ }_{1}$ in $\mathrm{NLME}^{5}$. Because the refinement of the linearization process is implemented in NLME, NLME is computationally faster than NONMEM.

In the study by Tokuda et $\mathrm{al}^{2}$, $\mathrm{t}_{1 / 2}$ estimated by model-independent analysis increased with the increase in dose. However, in this NLME analysis, the linear model yielded values in good agreement with observed values. The random effects on $t_{1 / 2}$ were very small in both one- and two-compartment analyses, i. e. $t_{1 / 2}$ was more or less similar in each patient. This might have occurred because the period of observation was not long enough to estimate $t_{1 / 2}$ correctly. If the linear model is appropriate for describing the disposition of trastuzumab, the previously reported dose-dependence of $t_{1 / 2}{ }^{2)}$ might be due to the truncated profile of the serum level used to calculate terminal $t_{1 / 2}$. In order to address this issue, data for multiple administration were analyzed.

\section{Multiple-dose administration}

Data for a total of 18 patients with metastatic breast cancer refractory to chemotherapy following multiple administration were fitted to one- and two-compartment models with zero-order infusion as shown in equations 1 and 2 . In these patients, a weekly dose of $1,2,4 \mathrm{or} 8 \mathrm{mg} / \mathrm{kg}$ of trastuzumab as a $90 \mathrm{~min}$ intravenous infusion was administered after the 21st day after initial administration. A 
Table 2 Summary of NLME Analysis of Multiple-administration Data using a Two-compartment Model

\begin{tabular}{|c|c|c|c|c|c|}
\hline \multicolumn{6}{|c|}{ Fixed effects: } \\
\hline & Value & Std.Error ${ }^{\text {a) }}$ & $\mathrm{DF}^{\mathrm{b})}$ & $\mathrm{t}$-value & $\mathrm{p}$-value \\
\hline $\mathrm{V}_{1}$ & 54.27090 & 2.428437 & 432 & 22.34808 & $<.0001$ \\
\hline$\alpha$ & 0.29045 & 0.042915 & 432 & 6.76813 & $<.0001$ \\
\hline$\beta$ & 0.04112 & 0.007577 & 432 & 5.42733 & $<.0001$ \\
\hline $\mathrm{k}_{21}$ & 0.12022 & 0.025446 & 432 & 4.72444 & $<.0001$ \\
\hline \multicolumn{6}{|c|}{ Random effects : } \\
\hline & $\mathrm{V}_{1}$ & $\alpha$ & $\beta$ & Residual & \\
\hline StdDev & 7.979612 & 0.00001096878 & 0.02204996 & 14820.86 & \\
\hline AIC & $\mathrm{BIC}$ & $\log \operatorname{Lik}$ & & & \\
\hline 10088.14 & 10121.07 & -5036.07 & & & \\
\hline \multicolumn{6}{|c|}{ Number of Observations : 453} \\
\hline \multicolumn{6}{|c|}{ Number of Groups : 18} \\
\hline
\end{tabular}

a) Parameter's standard error b) Degree of freedom

Table 3 Comparison of Mean of Individual $t_{1 / 2}$ (day) from NLME Analysis for Various Doses of Trastuzumab

\begin{tabular}{|c|c|c|c|c|c|}
\hline \multirow{2}{*}{$\begin{array}{c}\text { Dose } \\
(\mathrm{mg} / \mathrm{kg})\end{array}$} & \multirow{2}{*}{$\mathrm{n}$} & \multicolumn{2}{|c|}{ Single dose } & \multicolumn{2}{|c|}{ Multiple dose } \\
\hline & & 1-compartment & 2-compartment & 1-compartment & 2-compartment \\
\hline 1 & 5 & $4.62 \pm 0.04$ & $14.8 \pm 0.00$ & $5.58 \pm 0.58$ & $13.1 \pm 2.28$ \\
\hline 2 & 3 & $4.47 \pm 0.06$ & $14.8 \pm 0.00$ & $5.21 \pm 1.27$ & $12.2 \pm 3.61$ \\
\hline 4 & 3 & $4.60 \pm 0.54$ & $14.8 \pm 0.00$ & $10.9 \pm 4.57$ & $32.9 \pm 13.1$ \\
\hline 8 & 6 & $5.39 \pm 1.48$ & $14.8 \pm 0.00$ & $9.83 \pm 3.96$ & $27.6 \pm 11.5$ \\
\hline Mean* & 17 & 4.71 & 14.8 & 6.7 & 16.9 \\
\hline
\end{tabular}

comparison between values predicted by the twocompartment model and observed values is shown in Fig. 2. NLME fitting slightly underestimated the observed concentrations at higher doses. The AIC for the one-compartment model was 10254, and that for the two-compartment model was 10088. (Table 2). The two-compartment model was thus better than the one-compartment model for describing the disposition of trastuzumab $(p<0.0001)$ in the case of multiple administration as well. NLME fitting using the three-compartment model was not attempted because it had already failed to fit the single-dose data. The deviation of values predicted by the model from the observed data may be due to nonlinear pharmacokinetics of trastuzumab or incorrect estimation of the $t_{1 / 2}$ of trasutuzumab.
However, this deviation was only modest, and might thus not be clinically significant.

As shown in Table 3, in the two-compartment analysis the estimated $t_{1 / 2}$ was longer at higher doses than at lower doses. Thus, dose-dependent $t_{1 / 2}$ was observed with multiple administration of trastuzumab. The reason for dose-dependent $t_{1 / 2}$ has been unclear. The tumor load, i. e. tumor-associated clearance might be one of the reasons to explain it.

\section{Effect of shed antigen on pharmacokinetics of trastuzumab}

The mechanism of action of trastuzumab is shown in Fig. 3. Two mechanisms of action, i. e. antibody-dependent cellular cytotoxicity and downmodulation of proliferative signals have been re- 


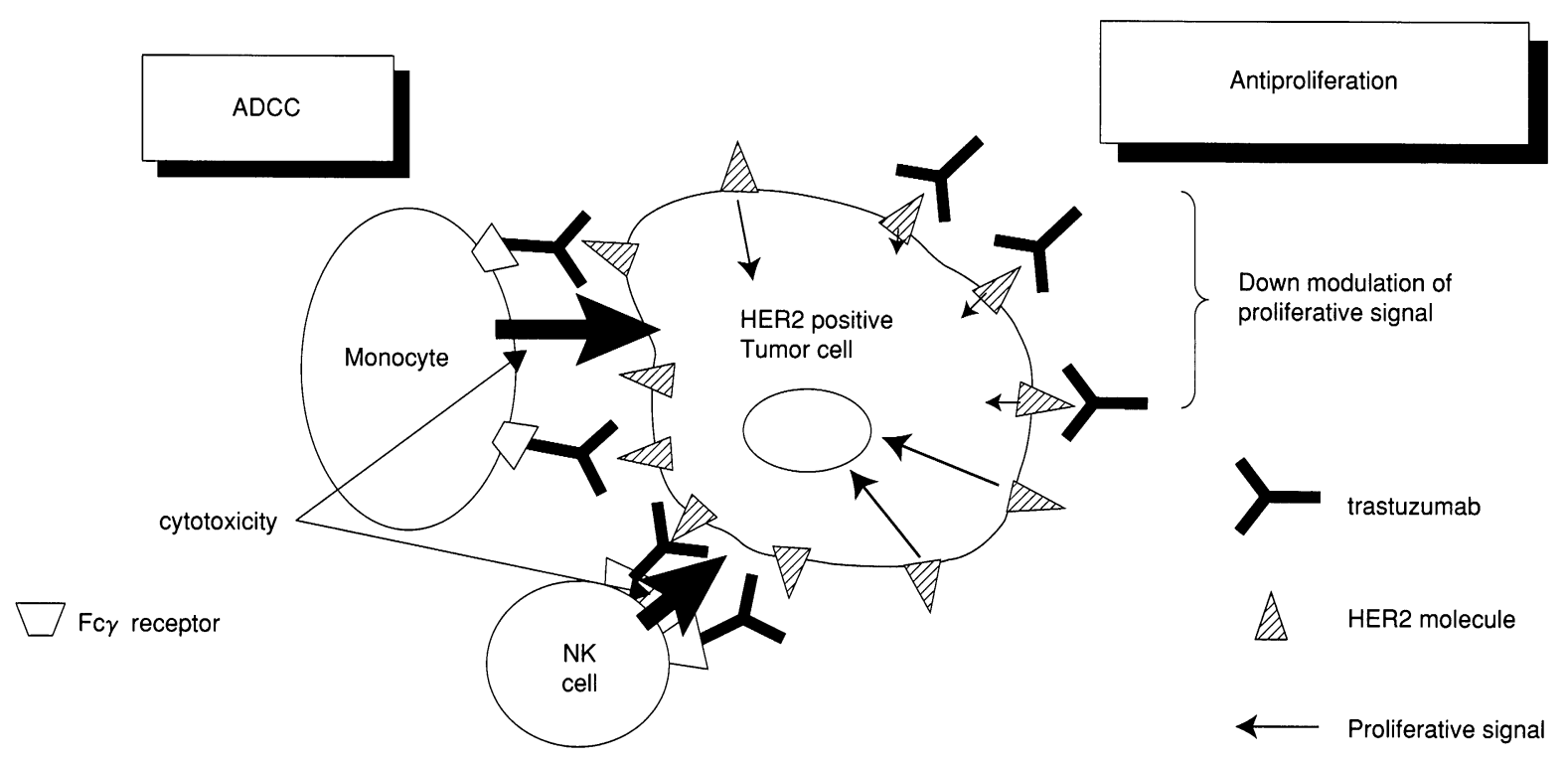

Fig. 3 The mechanism of action of tratuzumab

Table 4 Summary of NLME Analysis of Effect of Shed Antigen on Pharmacokinetics of Trastuzumab

\begin{tabular}{|c|c|c|c|c|c|}
\hline \multicolumn{6}{|l|}{ Fixed effects: } \\
\hline & Value & Std.Error ${ }^{\text {a) }}$ & $\mathrm{DF}^{\mathrm{b})}$ & $\mathrm{t}$-value & $\mathrm{p}$-value \\
\hline$\beta$ (Intercept) & 0.054 & 0.0172 & 200 & 3.132 & 0.0020 \\
\hline$\beta \mathrm{SHDAG}^{\mathrm{c})}$ & 0.000 & 0.0002 & 200 & 2.136 & 0.0339 \\
\hline $\mathrm{V}_{1}$ & 51.394 & 2.4303 & 200 & 21.147 & $<.0001$ \\
\hline$\alpha$ & 0.497 & 0.1623 & 200 & 3.063 & 0.0025 \\
\hline $\mathrm{k}_{21}$ & 0.292 & 0.1408 & 200 & 2.071 & 0.0396 \\
\hline \multicolumn{6}{|c|}{ Random effects : } \\
\hline & $\mathrm{V}_{1}$ & $\alpha$ & $\beta$ & \multicolumn{2}{|c|}{ Residual } \\
\hline StdDev & 7.1014 & 0.00013181 & 0.000022454 & \multicolumn{2}{|c|}{13046} \\
\hline AIC & $\mathrm{BIC}$ & $\log \mathrm{Lik}$ & & & \\
\hline 4878.5 & 4909.1 & -2430.2 & & & \\
\hline \multicolumn{6}{|c|}{ Number of Observations : 222} \\
\hline \multicolumn{6}{|c|}{ Number of Groups : 18} \\
\hline
\end{tabular}

ported. Like many other cell surface receptors, a soluble form of the extracellular domain of HER2 (shed antigen) can be shed from the surface of tumor cells and is detectable in the sera of approximately $20 \%$ to $25 \%$ of patients with locally advanced or metastatic breast cancer ${ }^{10)}$. Trastuzumab binds to shed antigen, resulting in antigen/ antibody complex formation that in turn results in more rapid clearance of trastuzumab from serum.
Therefore, the effect of shed antigen on the pharmacokinetics of trastuzumab was studied, with shed antigen used as a covariate for beta in the two-compartment model. As shown in Table 4, the effect of shed antigen on the $\beta$-phase slope was modest. Thus, the effect of shed antigen on the pharmacokinetics of trastuzumab was concluded to be insignificant in clinical practice. 

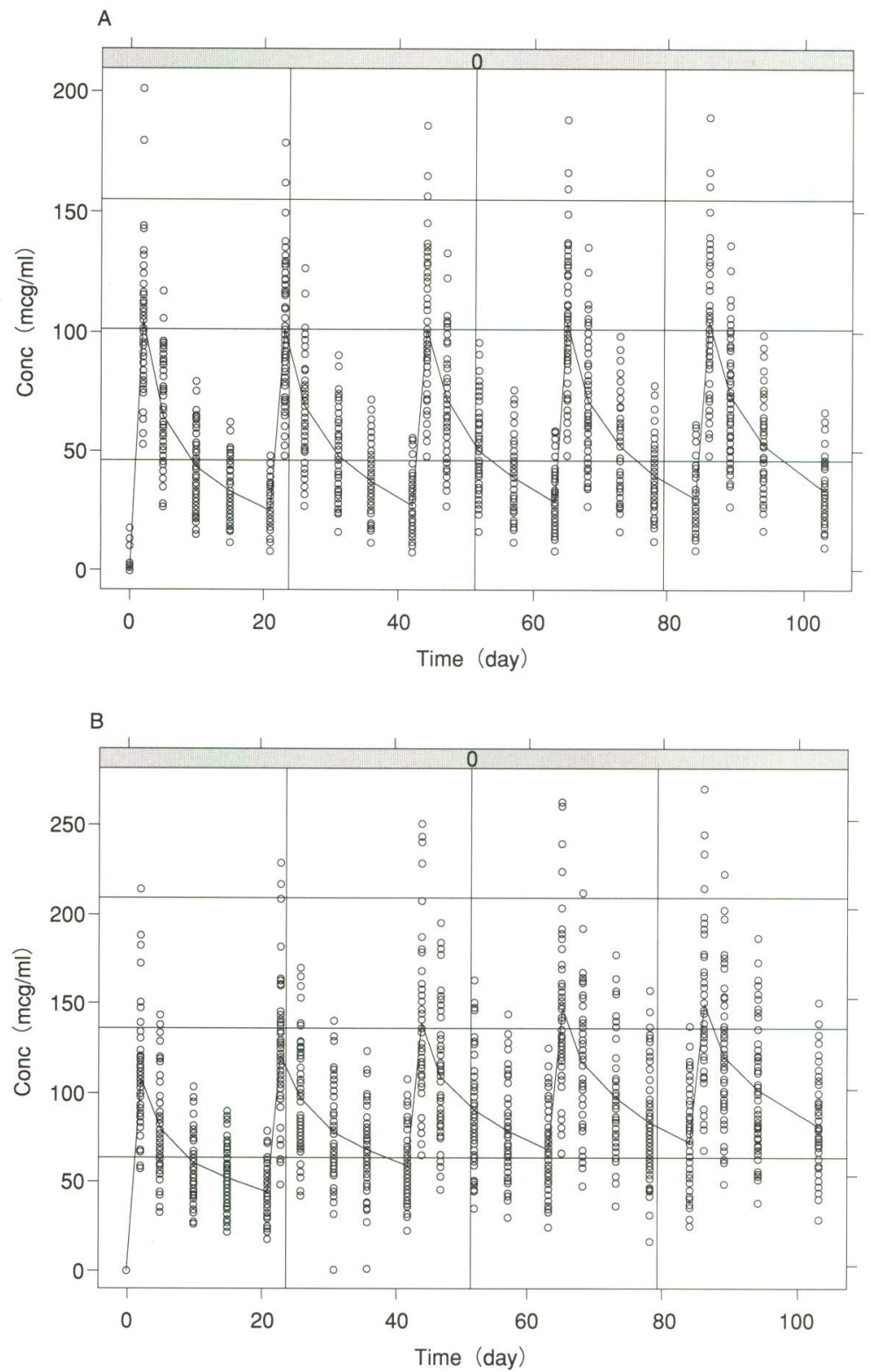

Fig. 4 Simulated serum levels of trastuzumab for 50 patients at a loading dose of $8 \mathrm{mg} /$ $\mathrm{kg}$ and a maintenance dose of $6 \mathrm{mg} / \mathrm{kg}$ once per 3 weeks. The pharmacokinetic parameters for the two-compartment model analysis of single-administration data $\left(A: t_{1 / 2}, 14.7\right.$ day) and multiple-administration data $\left(B: t_{1 / 2}, 27.6\right.$ day) were used in this simulation. The inter-subject variation of $20 \%$ was used for each pharmacokinetic parameter. The intra-subject variation of $10 \%$ was used for the serum level.

\section{Future usefulness of this modeling}

The use of pharmacokinetic/pharmacodynamic modeling and simulation in drug development has increased as evidenced by the increased presence in regulatory documents ${ }^{11}$. The simulation is useful to predict the outcome for a modified design. For the purpose of patient compliance in future adjuvant therapy, serum levels of trastuzumab at a loading dose of $8 \mathrm{mg} / \mathrm{kg}$ and maintenance dose of $6 \mathrm{mg} / \mathrm{kg}$ once per 3 weeks were simulated using the pharmacokinetic parameters obtained in the twocompartment analysis for single-dose data. Given the possibility of dose-dependency of $t_{1 / 2}$ for trastuzumab, the $t_{1 / 2}$ of 27.6 days obtained for multiple 
administration of $8 \mathrm{mg} / \mathrm{kg}$ was also used. The simulated trough serum levels were more or less similar to the observed values of $2 \mathrm{mg} / \mathrm{kg}$ once per week dose as shown in Fig. 4. It can thus be expected that the efficacy and safety at a loading dose of $8 \mathrm{mg} / \mathrm{kg}$ with maintenance dose of $6 \mathrm{mg} / \mathrm{kg}$ once per 3 weeks are similar to those for a $2 \mathrm{mg} / \mathrm{kg}$ once per week dose.

\section{Conclusion}

The two-compartment model was better than the one-compartment model in describing the disposition of trastuzumab, for both single and multiple administration. In single-dose fitting, the predicted concentrations agreed well with the observed concentrations in the one-compartment model as well as the two-compartment model. However, in multiple-dose fitting, the predicted concentrations slightly underestimated the observed concentrations at higher doses; this was considered to possibly be due to the nonlinear pharmacokinetics of trastuzumab or the problems with the estimation of half-life.

\section{Acknowledgements}

We thank Dr. Sarah Marston, Pharma StatSci, Arlington, $\mathrm{VA}$, for helpful S programming.

\section{References}

1) Olson, S. C., Bockbrader, H., Boyd, R. A., et al. : Impact of population pharmacokinetic-pharmacodynamic analyses on the drug development process. Clin. Pharmacokinet., 38 : 449-459 (2000).

2) Tokuda, Y., Watanabe, T., Omuro, Y., et al. : Dose escalation and pharmacokinetic study of a humanized anti-HER2 monoclonal antibody in patients with HER2/neuoverexpressing metastatic breast cancer. Br. J. Cancer, 81 : 1419-1425 (1999).

3) Aarons, L. : Software for population pharmacokinetics and pharmacodynamics. Clin. Pharmacokinet., 36 : 255-264 (1999).

4) Ette, E. I., Williams, P., Fadiran, E., et al. : The process of knowledge discovery from large pharmacokinetic data sets. J. Clin. Pharmacol., $41: 25-34$ (2001).

5) Pinheiro, J. C. and Bates, D. M. : Mixed-Effects Models in S and S-PLUS, Springer-Verlag, Inc, New York (2000).

6) MathSoft Inc. Seattle, WA. S-PLUS 2000 Release 3 (2000).

7) Jonsson, E. N. and Karlsson, M. O. : "Xpose - an S-PLUS based population pharmacokinetic/pharmacodynamic model building aid for NONMEM". Comp. Meth. Prog. Biomed., 58 : 51-64 (1999).

8) Pharma Stat Sci. S pklibrary, version alpha 1 (2001). URL : http://www.pharmastatsci.com/pklibrary.htm

9) Microsoft Co. Redmond, WA. Visual C++ 6.0 (1998).

10) Yamauchi, H., O'Neill, A., Gelman, R., et al. : Prediction of response to antiestrogen therapy in advanced breast cancer patients by pretreatment circulating levels of extracellular domain of the HER-2/c-neu protein. J. Clin. Oncol., 15: 2518-2525 (1997).

11) Aarons, L., Karlsson M. O., Mentré, F., et al. : Role of modelling and simulation in Phase I drug development. Eur. J. Pharm. Sci., 13:115-122 (2001). 


\section{APPENDIX}

\section{C source code for zero-order infusion two-compartment model}

S-plus scripts can be obtained by e-mail to tomoo.funaki@roche.com.

/* Zero-order input model Visual C */

\#include $<$ S. h $>$

$\#$ \#include <newredef. h>

\#define longint long int

$/ *$ One Compartment IV zero-order input */

void oneC_IVinf_macro (long int ${ }^{*}$ nrow, double $*$ Resp, double *inmat) \{

long int $\mathrm{i}, \mathrm{j}, \mathrm{nn}={ }^{*}$ nrow, $\mathrm{mm}=0$;

double $\mathrm{v}$, beta, $\mathrm{k} 0, \mathrm{~b}, \mathrm{tstar}, *_{\mathrm{t}} \mathrm{l}=$ Calloc $(\mathrm{nn}, \operatorname{double}), *_{\mathrm{t}} \mathrm{i}=$ Calloc (nn, double), $*$ ds $=$ Calloc (nn, double), *Subject, *Time, *Tinf, *Dose, *V, *Beta,

$\mathrm{sl}=$ DOUBLE_EPS; $\quad{ }^{*} \mathrm{sl}$ is last subject number, usually */

$/^{*}$ an integer but passed as double. */

Subject $=$ inmat

$/ *$ It is started at an unlikely value. */

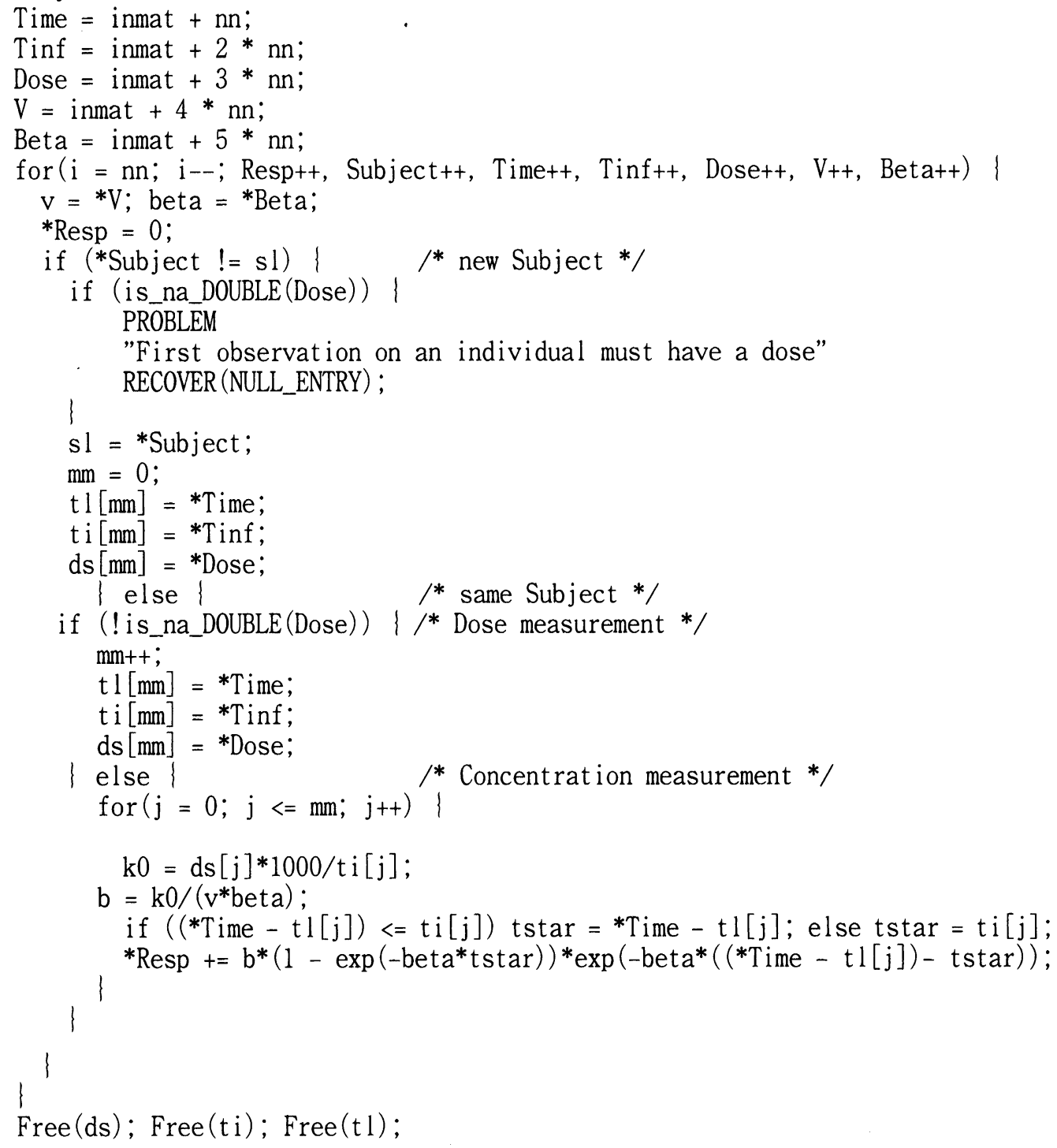




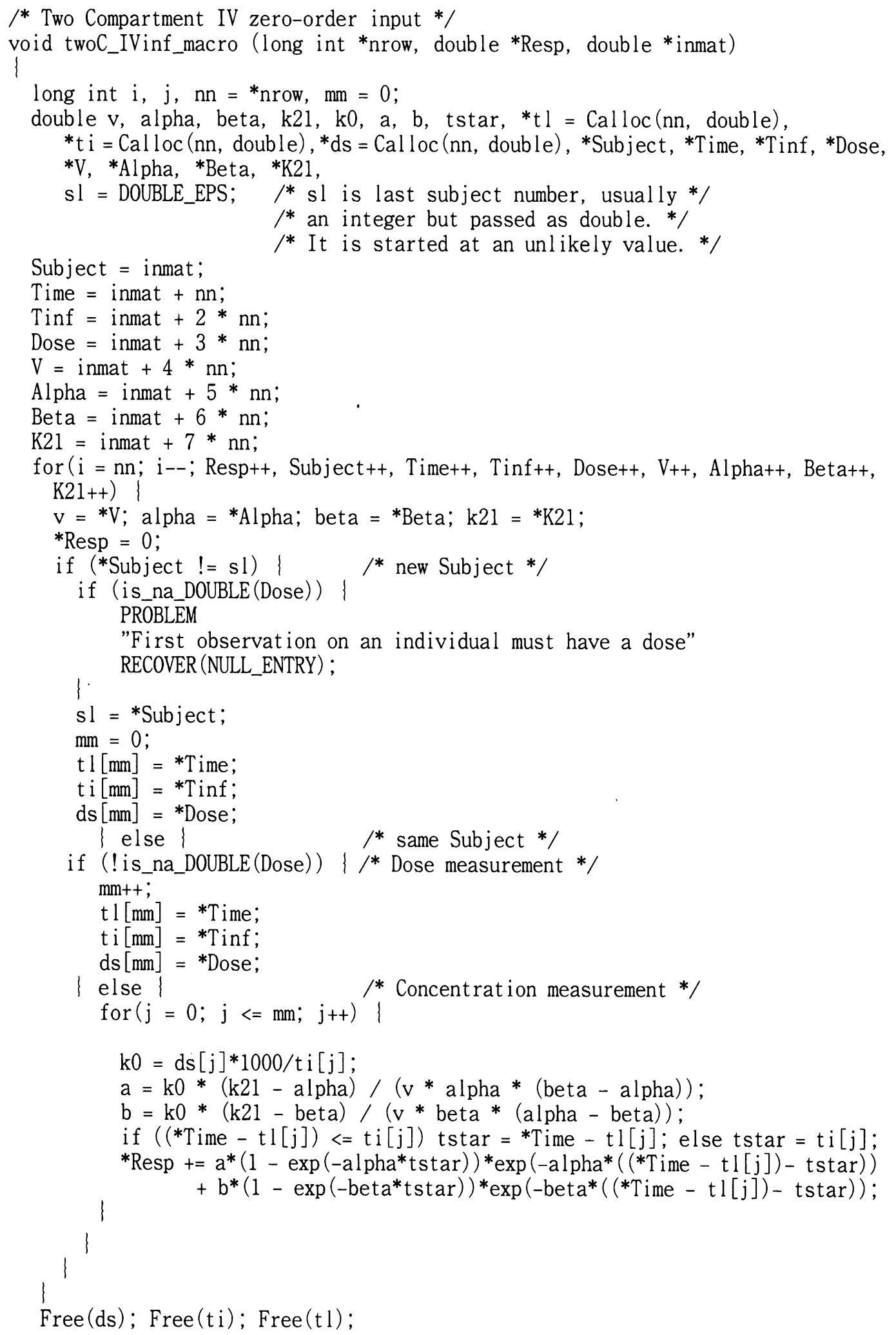

